\title{
Validity of modified gastrectomy combined with sentinel node navigation surgery for early gastric cancer
}

\author{
Hironori Ohdaira, Hiroshi Nimura, Norio Mitsumori, Naoto Takahashi, Hideyuki Kashiwagi, \\ and Katsuhiko Yanaga \\ Department of Surgery, The Jikei University School of Medicine, 3-25-8 Nishi-shinbashi, Minato-ku, Tokyo 105-8461, Japan
}

\begin{abstract}
Background. The present study examined the clinical validity of modified gastrectomy for early gastric cancer, in terms of the results of sentinel node navigation surgery (SNNS), using infrared ray electronic endoscopy (IREE) plus indocyanine green (ICG) staining.

Methods. One-hundred and sixty-one patients with fT1N0 gastric cancer were enrolled in the study. ICG $(0.5 \mathrm{ml}, 5 \mathrm{mg} /$ ml) was injected endoscopically into four quadrants of the submucosa surrounding the cancer. Twenty minutes after the injection, sentinel lymph nodes (SNs) stained with ICG were observed intraperitoneally around the serosa and surrounding fat tissue. IREE was used to illuminate regional lymph nodes from the serosal side.

Results. Group 2 lymph nodes were judged as SNs in 52 patients $(32 \%)$. The most common locations of the SNs were stations No. 7 in each of the upper-, middle-, and lower-thirds of the stomach. In two patients, lymph node metastasis was positive. One of these patients, with cancer in the middle onethird of the stomach, had SNs in stations No. 3, 4sb, 4d, 7, and No. 11p, and had metastatic lymph nodes in No. 3 and No. 7 (all SNs). The other patient, with cancer in the lower one-third of the stomach, had SNs in No. 1, 3, 4d, and 6, and had a metastatic lymph node in No. 4d (SN). Skip metastasis was not observed in this study, and metastatic lymph nodes were judged to have been dissected by the D1 $+\alpha$ procedure.

Conclusion. For T1N0 gastric cancer, modified gastrectomy (D1 $+\alpha$ dissection) combined with SNNS is suitable; however, for those whose Group 2 lymph nodes are judged to be SNs, additional dissection of lymphatic basins detected by SNNS should be performed to confirm the absence of lymph node metastasis.
\end{abstract}

Key words Modified gastrectomy · Sentinel node navigation surgery · Group 2 lymph nodes

Offprint requests to: $\mathrm{H}$. Ohdaira

Received: January 23, 2007 / Accepted: April 10, 2007

\section{Introduction}

In a study by Bonenkamp et al. [1], patients with gastric cancer treated by extended (D2) lymphadenectomy had a significantly higher rate of complications, a higher postoperative mortality rate and a longer hospital stay than those who had limited (D1) dissection, although 5 -year survival rates were similar in the two groups. Unnecessary extended lymphadenectomy should therefore be avoided to reduce the incidence of complications.

Recently, much attention has been paid to the improvement of postoperative function and quality of life after gastrectomy for early gastric cancer without impairing long-term outcome. However, several studies have shown features of skip metastasis in gastric, as well as in other cancers [2-4]. Because minimally invasive surgery has been applied to gastric cancer, the occurrence of skip metastasis is an important issue.

According to the treatment guidelines of the Japanese Gastric Cancer Association (JGCA), endoscopic mucosal resection (EMR) or modified gastrectomy (modified A, D1 + No. 7; modified B, D1 + No. 7, 8a, 9) is indicated for stage IA (T1N0) disease [5].

During the past several years, in patients with cT1N0 or cT2N0 early gastric cancer, we have performed sentinel node navigation surgery (SNNS), using infrared ray electronic endoscopy (IREE) combined with indocyanine green (ICG) injection [6]. With this method, we have encountered patients for whom Group 2 lymph nodes were judged as the sentinel lymph nodes (SNs). For such patients, we should be cautious about the extent of lymph node dissection.

In the present study, we examined the clinical validity of modified gastrectomy for early gastric cancer, in terms of the results of SNNS, using IREE combined with ICG staining. 


\section{Patients and methods}

The study protocol was approved by the Ethics Committee for Biomedical Research of the Jikei Institutional Review Board, and all patients provided informed consent. Patients admitted to Jikei University Hospital with gastric cancer with no obvious metastasis were enrolled prospectively in the study, and cT1N0 or cT2N0 gastric cancer patients who gave informed consent underwent SNNS. In this study, 161 patients with fT1N0 $(\mathrm{pT} 1 \mathrm{pN} 0)$ disease were evaluated. Patients who had lymph node metastasis diagnosed by preoperative abdominal computed tomography or ultrasonography; and those with $\mathrm{T} 2$ subserosal or $\mathrm{T} 3$ lesions (according to the tumor node metastasis [TNM] classification system), confirmed by endoscopic ultrasonography, were excluded. SNNS was performed according to the methods of Nimura et al. [6]. Briefly, $0.5 \mathrm{ml}$ ICG $(5 \mathrm{mg} / \mathrm{ml}$; Diagnogreen; Daiichi Pharmaceutical, Tokyo, Japan) was injected endoscopically in four quadrants of the submucosa surrounding the gastric cancer, using an endoscopic puncture needle during open or laparoscopic surgery. Twenty minutes after the injection, SNs stained with ICG were observed intraperitoneally around the serosa and surrounding fat tissue. IREE (Olympus Optical, Tokyo, Japan) was used to illuminate regional lymph nodes from the serosal side. Positive staining was confirmed by at least four surgeons and an endoscopist during surgery (Fig. 1). If possible, suspected SNs were examined by frozen section, with hematoxylin-andeosin staining, to determine the pathological diagnosis. Lymph node dissection and gastrectomy were performed according to the criteria of the gastric cancer treatment guidelines of the JGCA, followed by a definitive pathological examination that included hematoxylin-and-eosin staining, and immunohistochemical staining with anticytokeratin antibody (CAM 5.2; Becton Dickinson, San Jose, CA, USA).
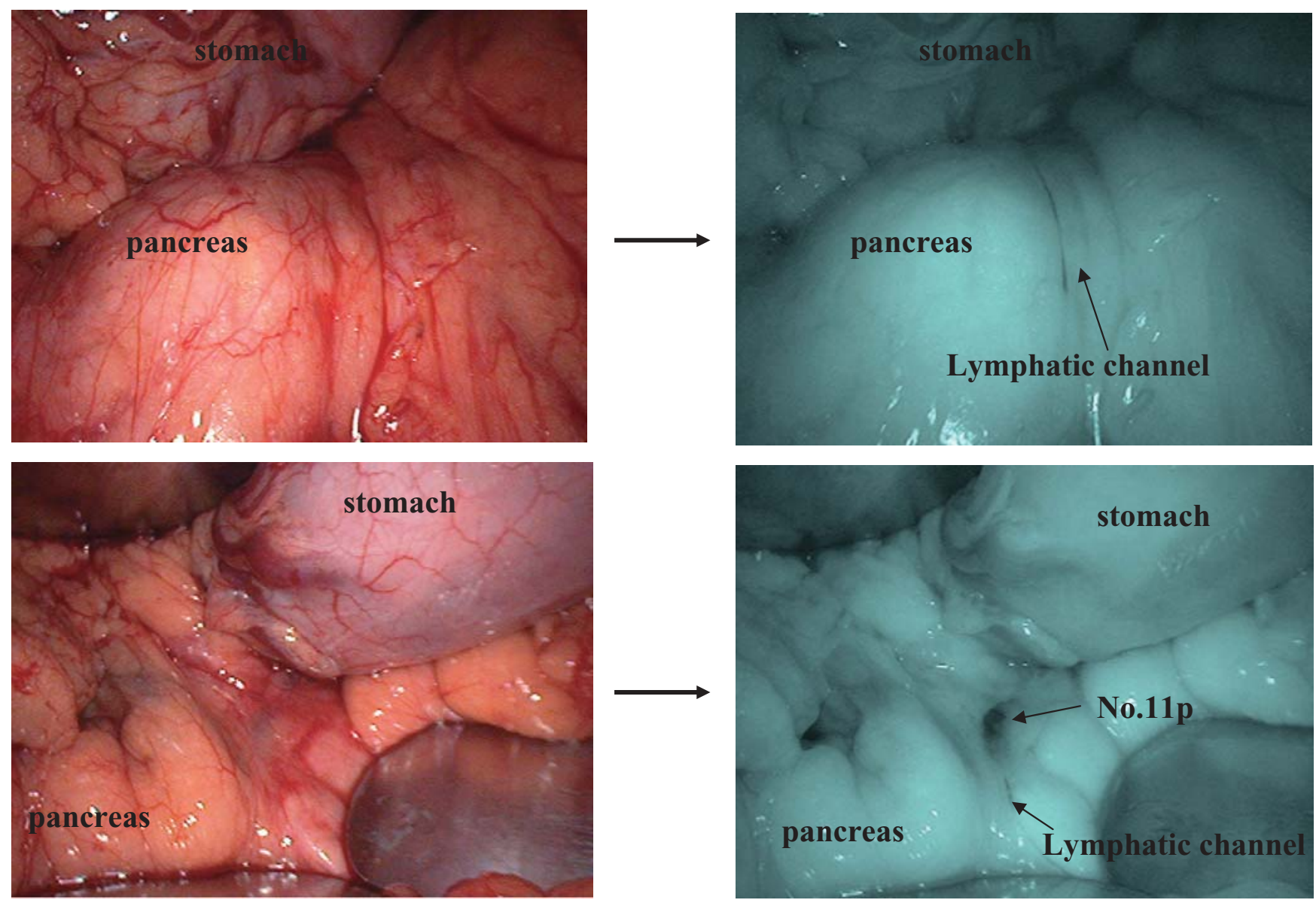

Ordinary light

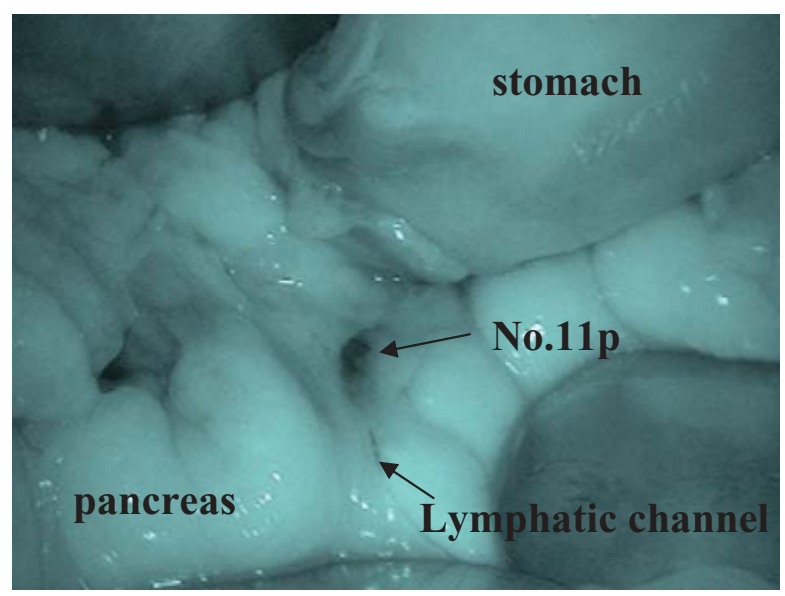

IREE

Fig. 1. Lymphatics stemming from the carcinoma. Lymphatic channels from the tumor follow a path crossing the anterior surface of the pancreas, revealing green-stained sentinel nodes (station No. 11p). IREE, infrared ray electronic endoscopy 


\section{Results}

Group 2 lymph nodes were judged as SNs in 52 patients (32\%; Table 1). Distal gastrectomy was performed in almost two-thirds of the 52 patients and the extent of lymph node dissection was $\mathrm{D} 1+\alpha$ in $58 \%$ of these patients.

The most common lymphatic basin of the cancer in the upper one-third of the stomach was the left gastric artery area (eight patients), while the most common location of SNs in cancer in the upper one-third of the stomach was station No. 7 (seven patients; Table 2). However, one patient had an $\mathrm{SN}$ in No. $4 \mathrm{~d}$.

For cancer in the middle one-third of the stomach, mainly the lymphatic compartments around both the left gastric artery and the right gastric artery were stained. Thirty-one of these patients had SNs in the No. 7 area, but in 7 patients, staining with ICG was seen in No. $9,11 \mathrm{p}, 12 \mathrm{a}$, or $14 \mathrm{v}$.

Gastric cancers in the lower one-third of the stomach predominantly drained to the right side, around the right gastroepiploic artery and the right gastric artery. Common locations of SNs were stations No. 7 and No. 8 a (11 patients). However, SNs were also observed in the areas of No. 1, 11p, and $14 \mathrm{v}$.

Lymphatic basins positive for ICG, in relation to the cancer location, are shown in Table 2. Only two patients had no drainage to the left gastric artery area. For cancers in the middle and lower one-thirds of the stomach, the most common drainage in the lymphatic basins was to the left gastric artery and the right gastroepiploic artery areas.

Two patients had lymph node metastasis (Fig. 2). Case 1 had a $45 \times 23-\mathrm{mm}$ submucosal signet ring cell carcinoma in the greater curvature of the middle onethird of the stomach, which had three lymphatic basins (left gastric artery, right gastric artery, and right gastroepiploic artery areas) and SNs in No. 3, 4sb, 4d, 7, and 11p. Metastatic lymph nodes were located in No. 3 and No. 7, which were all SNs. Group 2 lymph nodes, No. $8 \mathrm{a}$ and No. $11 \mathrm{p}$, were dissected. Case 2 had a $23 \times 12-\mathrm{mm}$ submucosal signet ring cell carcinoma in the anterior wall of the lower one-third of the stomach, which had two lymphatic basins (left gastric artery and right gastroepiploic artery areas) and SNs in No. 1, 3, 4d, and 6. A metastatic lymph node was located in No. $4 \mathrm{~d}$, and this was an $\mathrm{SN}$. D1 $+\alpha$ resection was performed.

Table 1. Characteristics of patients with Group 2 lymph nodes judged to be SNs

\begin{tabular}{lc}
\hline Age (years) & $59.7 \pm 10.0$ (35-76) \\
Sex ratio (M:F) & $37: 15$ \\
Tumor location & 7 \\
$\quad$ Upper one-third & 33 \\
Middle one-third & 12 \\
$\quad$ Lower one-third & \\
Extent of resection & 2 \\
$\quad$ Proximal gastrectomy & 33 \\
Distal gastrectomy & 4 \\
Total gastrectomy & 5 \\
Pylorus-preserving gastrectomy & 5 \\
Segmental gastrectomy & 3 \\
Wedge resection & \\
Extent of dissection & 30 \\
D1+ $\alpha$ & 6 \\
D1+ $\beta$ & 9 \\
D2 & 7 \\
LBD & \\
\hline
\end{tabular}

LBD, Lymphatic basin dissection; SN, sentinel lymph node

Table 2. Lymphatic basins positive for ICG, in relation to the cancer location

\begin{tabular}{llrlr}
\hline Location of tumor & \multicolumn{1}{c}{ Lymphatic basin } & \multicolumn{2}{c}{$\begin{array}{c}\text { Location } \\
\text { of SNs }\end{array}$} \\
\hline Upper one-third & LGA alone & 4 & No. 7 & 7 \\
& LGA and PGA & 2 & No. 4d & 1 \\
& LGA, LGEA, and PGA & 1 & & \\
Middle one-third & LGA, LGEA, and RGEA & 1 & & 31 \\
& LGA alone & 11 & No. 7 & 4 \\
& LGA and RGEA & 16 & No. 8a & 1 \\
& LGA and RGA & 2 & No. 9 & 4 \\
& LGA, LGEA, and RGEA & 1 & No. 11p & 1 \\
& LGA, RGEA, and RGA & 1 & No. 12a & 1 \\
& LGA, LGEA, RGEA, and RGA & 1 & No. 14v & 6 \\
& LGA alone & 1 & No. 7 & 5 \\
& RGE alone & 2 & No. 8a & 2 \\
& LGA and RGEA & 2 & No. 1 & 1 \\
& LGA and RGA & 1 & No. 11p & 3 \\
& RGA and RGEA & 2 & No. 14v & 3 \\
& LGA, RGA, and RGEA & 4 & & \\
\hline
\end{tabular}

LGA, Left gastric artery; RGA, right gastric artery; PGA, posterior gastric artery; LGEA, left gastroepiploic artery; RGEA, right gastroepiploic artery 


\section{Case 1}

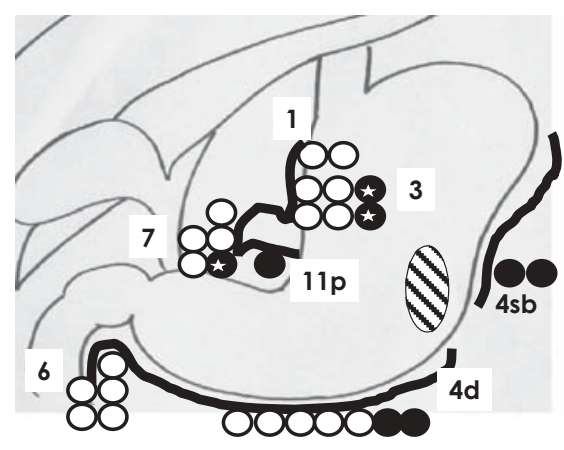

Case 2

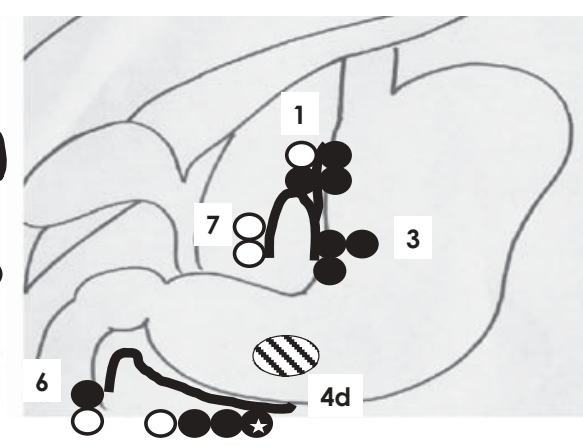

Location of gastric cancer

Non-sentinel lymph node

Sentinel lymph node

Metastatic sentinel lymph node

Lymphatic basin stained by ICG
Fig. 2. Details of the two patients who had metastatic lymph nodes. Case 1 had a $45 \times 23-\mathrm{mm}$ submucosal signet ring cell carcinoma in the greater curvature of the middle one-third of the stomach, which had three lymphatic basins, in the left gastric artery (LGA), right gastric artery (RGA), and right gastroepiploic artery (RGEA) areas, and sentinel lymph nodes (SNs) in stations No. 3, 4sb, 4d, 7, and 11p. Metastatic lymph nodes were located in No. 3 and No. 7; these were all SNs (n2 [3/29] No. 1, 0/2; No. 3, 2/6; No. 4sb, 0/2; No. 4 d, $0 / 7$; No. $5,0 / 1$; No. $6,0 / 5$; No. 7 , $1 / 5$; No. 11 p, 0/1). Case 2 had a $23 \times 12$ $\mathrm{mm}$ submucosal signet ring cell carcinoma in the anterior wall of the lower one-third of the stomach, which had two lymphatic basins (LGA and RGEA) and SNs in No. $1,3,4 \mathrm{~d}$, and 6 . A metastatic lymph node was located in No. 4d, and this was an SN (n1 [1/16] No. 1, 0/4; No. 3, 0/3; No. 4d, 1/4; No. 6, 0/2; No. 7, 0/2; No. 8a, 0/1). $I G C$, indocyanine green
All the SNs in Group 2 lymph nodes were detected with IREE plus ICG. Skip metastasis was not observed, and metastatic lymph nodes were judged to have been dissected by the $\mathrm{D} 1+\alpha$ procedure. There were no cases of postoperative metastasis or recurrence.

\section{Discussion}

According to the treatment guidelines of the JGCA, endoscopic mucosal resection (EMR) or modified gastrectomy is indicated for stage IA (T1N0) disease as the routine practice [5]. In addition, local resection and segmental resection are proposed for clinical trials [5]. These recommendations are based on the large amounts of data obtained from patients who have undergone gastrectomy in Japan. Although the guidelines were developed based on as much evidence as possible, the guideline-developing committee did face difficulties because of the lack of evidence for various aspects of treatments [7].

During SNNS using ICG, we sometimes encountered SNs in the Group 2 lymph node area. These findings suggest that even early gastric cancer may be associated with metastasis to the Group 2 lymph node area, or skip metastasis. Therefore, we were faced with the following question: "Is D1+ $\alpha$ lymph node dissection really enough for T1N0 gastric cancer?"

As to the skip metastasis of gastric cancer, Park et al. [8] analyzed findings in 14 patients with such metastasis among 266 patients who had undergone more than D2 lymph node dissection. The incidence of skip metastasis was $5.3 \%$, and only 1 patient with early gastric cancer had such metastasis. The lymph node stations of the skip metastasis were No. 7, 8a, 9, 11p, and No. 1. The survival rate did not show any statistically significant difference between those with and those without skip metastasis. Park et al. [8] concluded that D2 lymph node dissection should be performed until sentinel lymph node (SN) detection became feasible and reliable, and that the potential risk from skip metastasis was not great, and therefore skip metastasis itself should not be a major consideration in therapeutic decisions.

Kikuchi et al. [9] analyzed the topographical pattern of lymph node metastasis for $\mathrm{pN} 1$ patients with curative resection. Skip metastasis occurred in 5\%, and the common stations for such a metastasis were No. 7 and No. $8 \mathrm{a}$. This pattern of metastasis was found in $14 \%$ of the patients with single positive nodes. Kikuchi et al. [9] noted that although perigastric nodes were important first sites of drainage, the distribution of positive nodes depended on the tumor location.

Accordingly, in view of both the complexity of the lymphatic circulation and skip metastasis, previous studies have recommended the routine use of systemic D2 dissection [9-11]. However, the feasibility and reliability of SNNS is a prerequisite for limited gastric resection [9-11].

In western countries, Roviello et al. [12], in their multicenter retrospective study, confirmed nodal involvement to be a significant prognostic factor for early gastric cancer. In view of the trend to a lower risk of recurrence when more than 15 nodes were retrieved and the better staging achieved, they concluded that D2 
lymphadenectomy was the treatment of choice. However, Degiuli et al. [13], in their retrospective analysis, reported that the survival benefit of $\mathrm{D} 2$ gastrectomy for early gastric cancer was not documented either in the overall population or in subset analyses of patients with increased risk of nodal metastasis.

Recently, reports on SNNS for gastric cancer have increased, in which technical improvements have been documented [14-20]. Using infrared ray electronic endoscopy (IREE), we previously reported a sensitivity of $100 \%$, specificity of $67 \%$, positive predictive value of $29 \%$, and negative predictive value of $100 \%$ for the detection of SNs [6].

In the present study, common locations of SNs were stations No. 7 and No. 8a. During the SNNS, if these lymph nodes were stained green, we were afraid that they may have been the stations of skip metastasis, as reported by others $[9,10]$. Interestingly, in the middleand lower-thirds of the stomach, stations No. 11p and No. $14 \mathrm{v}$ (which are necessary to include for more than D2 dissection) were included as SNs. However, metastatic lymph nodes were located in stations No. 3 and No. 7 in one patient, and in No. $4 \mathrm{~d}$ in another patient. They were all SNs. Furthermore, skip metastasis were not observed and metastatic lymph nodes were considered to have been dissected by the $\mathrm{D} 1+\alpha$ procedure.

In T1N0 stage disease, the first sites of metastasis are the perigastric nodes, and skip metastasis seems to occur rarely. In previous studies, almost all patients with skip metastasis had advanced cancer $[8,9,10,11]$. As mentioned previously, in the study by Park et al. [8], only one patient with early gastric cancer had skip metastasis.

As to the lymphatic basins that were positive for ICG, we found that the left gastric artery compartment was the most common area, regardless of the location of the cancer. Furthermore, as many as $61.5 \%$ of the patients in our study had more than one lymphatic basin positive for ICG, including the left gastric artery area (mainly the left gastric artery and right gastroepiploic artery areas).

Miwa et al. [20] advocated the concept of lymphatic basin dissection with SNNS, using patent blue as a tracer. The sensitivity and accuracy of their method were $85 \%$ and $98 \%$, respectively. They reported that $\mathrm{T} 1$ gastric cancer involved a single lymphatic basin in $42 \%$ of their patients with gastric cancer, two lymphatic basins in $47 \%$, and three in $12 \%$. Similar to findings in our study, they found that the most common drainage lymphatic basins were the left gastric artery and right gastroepiploic artery areas, especially for cancers in the middle and lower one-thirds of the stomach. In their series, patients with one or two basins were treated with limited gastric resection with en-bloc dissection of the blue lymphatic basins, and none developed recurrence or died of cancer.

If SNs are detected in Group 2 lymph nodes during modified gastrectomy (such as local resection, segmental gastrectomy, pylorus-preserving gastrectomy, or distal as well as proximal gastrectomy), dissection of the lymphatic basin positive for ICG would identify metastatic lymph nodes. In patients in whom the lymphatic basin positive for ICG includes the left gastric artery area, No. 7 is the most important lymph node station.

Therefore, for patients with T1N0 gastric cancer, modified gastrectomy (D1+ $\alpha$ dissection), combined with SNNS, is suitable; however, for those whose Group 2 lymph nodes have been judged as SNs, additional dissection of the ICG-positive lymphatic basin, detected by SNNS, should be performed to confirm the absence of lymph node metastasis.

\section{References}

1. Bonenkamp JJ, Hermans J, Sasako M, van de Velde CJ, Welvaart K, Songun I, et al; Dutch Gastric Cancer Group. Extended lymphnode dissection for gastric cancer. N Engl J Med 1999;340: 908-14.

2. Prenzel KL, Monig SP, Sinning JM, Baldus SE, Gutschow CA, Grass G, et al. Role of skip metastasis to mediastinal lymph nodes in non-small cell lung cancer. J Surg Oncol 2003;82:25660.

3. Merrie AE, Phillips LV, Yun K, McCall JL. Skip metastases in colon cancer: assessment by lymph node mapping using molecular detection. Surgery 2001;129:684-91.

4. Rosen PP, Lesser ML, Kinne DW, Beattie EJ. Discontinuous or "skip" metastases in breast carcinoma. Analysis of 1228 axillary dissections. Ann Surg 1983;197:276-83.

5. Nakajima T. Gastric cancer treatment guidelines in Japan. Gastric Cancer 2002;5:1-5.

6. Nimura H, Narimiya N, Mitsumori N, Yamazaki Y, Yanaga K, Urashima M. Infrared ray electronic endoscopy combined with indocyanine green injection for detection of sentinel nodes of patients with gastric cancer. Br J Surg 2004;91:575-9.

7. Introduction to JGCA gastric cancer treatment guidelines. http://www.jgca.jp/PDFfiles/E-gudeline.PDF

8. Park SS, Ryu JS, Min BW, Kim WB, Kim SJ, Kim CS, et al. Impact of skip metastasis in gastric cancer. A N Z J Surg 2005; 75:645-9.

9. Kikuchi S, Kurita A, Natsuya K, Sakuramoto S, Kobayashi N, Shimao H, et al. First drainage lymph node(s) in gastric cancer: analysis of the topographical pattern of lymph node metastasis in patients with pN-1 stage tumors. Anticancer Res 2003;23:6014.

10. Ichikura T, Furuya Y, Tomimatsu S, Okusa Y, Ogawa T, Mukoda $\mathrm{K}$, et al. Relationship between nodal stage and the number of dissected perigastric nodes in gastric cancer. Surg Today 1998; 28:879-83.

11. Maruyama K, Gunven P, Okabayashi K, Sasako M, Kinoshita T. Lymph node metastases of gastric cancer. General pattern in 1931 patients. Ann Surg 1989;210:596-602.

12. Roviello F, Rossi S, Marrelli D, Pedrazzani C, Corso G, Vindigni $\mathrm{C}$, et al. Number of lymph node metastases and its prognostic significance in early gastric cancer: a multicenter Italian study. J Surg Oncol 2006;94:275-80. 
13. Degiuli M, Calvo F. Survival of early gastric cancer in a specialized European center. Which lymphadenectomy is necessary? World J Surg 2006;30:2193-203.

14. Kitagawa Y, Fujii H, Mukai M, Kubota T, Ando N, Ozawa S, et al. Intraoperative lymphatic mapping and sentinel lymph node sampling in esophageal and gastric cancer. Surg Oncol Clin N Am 2002;11:293-304.

15. Miwa K, Kinami S, Taniguchi K, Fushida S, Fujimura T, Nonomura A. Mapping sentinel nodes in patients with early-stage gastric carcinoma. Br J Surg 2003;90:178-82.

16. Kim MC, Jung GJ, Lee JH, Choi SR, Kang DY, Roh MS, et al. Sentinel lymph node biopsy with 99mTC tin-colloid in patients with gastric carcinoma. Hepatogastroenterology 2003;50(Suppl 2):ccxiv-ccxv.
17. Aikou T, Kitagawa Y, Kitajima M, Uenosono Y, Bilchik AJ, Martinez SR, et al. Sentinel lymph node mapping with GI cancer. Cancer Metastasis Rev 2006;25:269-77.

18. Isozaki H, Kimura T, Tanaka N, Satoh K, Matsumoto S, Ninomiya $\mathrm{M}$, et al. An assessment of the feasibility of sentinel lymph node-guided surgery for gastric cancer. Gastric Cancer 2004;7: 149-53.

19. Miyake K, Seshimo A, Kameoka S. Assessment of lymph node micrometastasis in early gastric cancer in relation to sentinel nodes. Gastric Cancer 2006;9:197-202.

20. Miwa K, Kinami S, Ajisaka H, Fushida S, Fujimura T. Lymphatic basin dissection and function-preserving limited gastrectomy for early-stage gastric carcinoma. Nippon Geka Gakkai Zasshi (J Jpn Surg Soc) 2005;106:280-5. 